Original Paper (Invited)

\title{
Thermal Effects on Cryogenic Cavitating Flows around an Axisymmetric Ogive
}

\author{
Suguo Shi, Guoyu Wang \\ School of Mechanical and Vehicular Engineering, \\ Beijing Institute of Technology, Beijing, 100081, China \\ shisuguo@bit.edu.cn,wangguoyu@bit.edu.cn
}

\begin{abstract}
Cavitation in cryogenic fluids generates substantial thermal effects and strong variations in fluid properties, which in turn alter the cavity characteristics. In order to investigate the cavitation characteristics in cryogenic fluids, numerical simulations are conducted around an axisymmetric ogive in liquid nitrogen and hydrogen respectively. The modified Merkle cavitation model and energy equation which accounts for the influence of cavitation are used, and variable thermal properties of the fluid are updated with software. A good agreement between the numerical results and experimental data are obtained. The results show that vapor production in cavitation extracts the latent heat of evaporation from the surrounding liquid, which decreases the local temperature, and hence the local vapor pressure in the vicinity of cavity becomes lower. The cavitation characteristics in cryogenic fluids are obtained that the cavity seems frothy and the cavitation intense is lower. It is also found that when the fluid is operating close to its critical temperature, thermal effects of cavitation are more obviously in cryogenic fluids. The thermal effect on cavitation in liquid hydrogen is more distinctively compared with that in liquid nitrogen due to the changes of density ratio, vapour pressure gradient and other variable properties of the fluid.
\end{abstract}

Keywords: cavitation; cryogenic fluid; thermal effect; numerical simulation; energy equation

\section{Introduction}

Cryogenic fluids, such as liquid hydrogen and oxygen, are often used as rocket propellants, and it is known that a suction performance of turbopump inducer in cryogenic fluid is improved due to "Thermal effect" ${ }^{\text {"[1] }}$. Thermal effect can be explained by temperature decrease inside a cavity region arising from the latent heat absorption. Long before in 1961, Sarosdy and Acosta ${ }^{[2]}$ detectes significant difference between water cavitation and Freon cavitation, and they obtain that while water cavitation is clear and more intense, cavitation in Freon, under similar conditions, is frothy with greater entrainment rates and lower intensity. Hord ${ }^{[3}$ publishes comprehensive experimental data on cryogenic cavitation in ogives. Pressure and temperature are measured at five probe locations over the geometries and several experiments are performed under varying inlet conditions. Their results are documented along with the instrumentation error. Franc ${ }^{[4]}$ adopts pressure spectra to investigate R-114 cavitation on inducer blades. The impact of thermal effect is examined at three reference fluid temperatures. They reports a delay in the onset of blade cavitation at higher reference fluid temperatures, which is attributed to suppression of cavitation by thermal effects. Although the experiments above-mensioned used different fluids and equipments, and the specific investigation contents is also not the same, there accurs obviously variety in the cavity size due to the thermal effect for all the experimental results. The mechanism of thermal effect on cavitation in cryogenic fluids have not been understood well compared with those of water at normal temperature because experimental approaches are not easy. For better understanding of thermal effects on cavitation, numerical simulation is one of the most important tools. From the point of the energy equation used or not, the numerical simulation development go through two stages. In the preliminary investigation, Stepanoff ${ }^{[5]}$, Ruggeri and Moore ${ }^{[6]}$ and Holl ${ }^{[7]}$ uses semi-empirical procedures to predict the thermal effect on cavitation. Despite the improvements in the approach, it is important to underscore the limitation that the above studies does not solve the energy equation in the domain. Ahuja ${ }^{[8]}$ and Utturkar ${ }^{[9]}$ recently ${ }^{[{ }}$ solve the energy equation in the entire domain with dynamic update of material properties and reported numerical studies on cavitation using liquid hydrogen and liquid nitrogen. For the former, their pressure and temperature predictions over a hydrofoil geometry is not obtained well with the experiment in the leading edge. And for the latter, the pressure and temperature predictions showed inconsistent agreement with the experimental data, especially at the cavity closure region. Cao ${ }^{[10]}$ validates Singhal

Accepted for publication November 30 2010: Paper number O10035S

Corresponding author: Wangguoyu, Professor, wangguoyu@bit.edu.cn.

This paper was presented at 2010 International Conference on Pumps and Fans, October 18-21, 2010, Hangzhou, China 
cavitation model in cryogenic cavitation flows and gets the pressure and temperature distribution, but the pressure is not obtained well with the experiment.

In the present study, the modified Merkle cavitation model and variable thermal properties of the fluid are realized via a UDF (user defined function) that is compiled and linked to the solver. The energy equation is used which considered the influence of cavitation. Validation calculations are carried out for an axisymmetric ogive for cryogenic fluids. According to these validation calculations, good agreement can be observed, and this result indicates that the numerical models are applicable for cryogenic fluids flow. Based on the numerical results, the cavity characteristics in cryogenic fluids are discussed.

\section{Physical and Numerical Model}

\subsection{Continuity and momentum equation}

The Navier-Stokes equations in their conservative form governing a Newtonian fluid without body forces and heat transfer and presented below in the Cartesian coordinates:

$$
\begin{gathered}
\frac{\partial \rho_{m}}{\partial t}+\frac{\partial\left(\rho_{m} u_{i}\right)}{\partial x_{i}}=0 \\
\frac{\partial\left(\rho_{m} u_{i}\right)}{\partial t}+\frac{\partial\left(\rho_{m} u_{i} u_{j}\right)}{\partial x_{j}}=-\frac{\partial p}{\partial x_{i}}+\frac{\partial}{\partial x_{j}}\left[\left(\mu+\mu_{t}\right)\left(\frac{\partial u_{i}}{\partial x_{j}}+\frac{\partial u_{j}}{\partial x_{i}}-\frac{2}{3} \frac{\partial u_{i}}{\partial x_{j}} \delta_{i j}\right)\right] \\
\frac{\partial}{\partial \mathrm{t}}\left[\rho_{m} C p T\right]+\frac{\partial}{\partial x_{j}}\left[\rho_{m} u_{j} C p T\right]=\frac{\partial}{\partial x_{j}}\left[\left(\frac{\mu}{\operatorname{Pr}_{L}}+\frac{\mu_{t}}{\operatorname{Pr}_{t}}\right) \frac{\partial h}{\partial x_{j}}\right]-\left\{\frac{\partial}{\partial t}\left[\rho_{m}\left(f_{v} L\right)\right]+\frac{\partial}{\partial x_{j}}\left[\rho_{m} u_{j}\left(f_{v} L\right)\right]\right\} \\
\rho_{m}=\rho_{l} \alpha_{l}+\rho_{v}\left(1-\alpha_{l}\right)
\end{gathered}
$$

\subsection{Turbulent model}

The $k-\omega$ SST turbulent ${ }^{[11]}$ equations are utilized to model the turbulence effect:

$$
\begin{gathered}
\frac{D \rho_{m} k}{D t}=P_{k}-\frac{\rho_{m} k^{3 / 2}}{l_{k-\omega}}+\frac{\partial}{\partial x_{i}}\left[\left(\mu+\frac{\mu_{t}}{\sigma_{k}}\right) \frac{\partial k}{\partial x_{i}}\right] \\
\frac{D \rho_{m} w}{D t}=C_{w} P_{w}-\beta_{w} \rho_{m} \omega^{2}+\frac{\partial}{\partial x_{i}}\left[\left(\mu+\frac{\mu_{t}}{\sigma_{k}}\right) \frac{\partial k}{\partial x_{i}}\right]+2 \rho_{m}\left(1-F_{1}\right) \sigma_{\omega 2} \frac{1}{\omega} \frac{\partial k}{\partial x_{i}} \frac{\partial \omega}{\partial x_{i}}
\end{gathered}
$$

The viscosity coefficient is defined as:

$$
v_{t}=\frac{a_{1} k}{\max \left(a_{1} \omega ; \Omega\right)}
$$

Here, the constant model number is got by reference ${ }^{[12]}$.

\subsection{Merkle $^{[13]}$ cavitation model}

$$
\begin{gathered}
\frac{\partial \alpha_{l}}{\partial t}+\nabla \cdot\left(\alpha_{l} \vec{u}\right)=\dot{m}^{+}-\dot{m}^{-} \\
\dot{m}^{-}=\frac{C_{\text {dest }} \rho_{v} M I N\left(p-p_{v}, 0\right) \alpha_{l}}{\left(0.5 \rho_{l} U_{\infty}^{2}\right) \rho_{v} t_{\infty}} \\
\dot{m}^{+}=\frac{C_{p r o d} \rho_{v} M A X\left(p-p_{v}, 0\right)\left(1-\alpha_{l}\right)}{\left(0.5 \rho_{l} U_{\infty}^{2}\right) t_{\infty}}
\end{gathered}
$$

As experimental investigations have shown significant effect of turbulence on cavitating flows, $p_{v}$ is set to be higher than the saturation pressure as follows ${ }^{[14]}$ :

$$
\begin{gathered}
p_{\text {turb }}=0.39 \rho k \\
p_{v}=\left(p_{v}\left(T_{l}\right)+p_{\text {turb }} / 2\right)
\end{gathered}
$$




\subsection{The boundary conditions and mesh}

We perform computations on the geometry experimentally investigated by $\operatorname{Hord}^{[3]}$, namely, an axisymmetric ogive. The geometry was mounted inside suitably designed tunnels in the experimental setup ${ }^{[3]}$. The computational domain is shown in Fig.1. The boundary conditions are implemented by stipulating the values of the velocity components (obtained from the experimental data), phase fraction, temperature, and turbulence quantities at the inlet. Furthermore, at the walls, the no-slip and adiabatic condition are extrapolated. Pressure and other variables are extrapolated at the outlet boundaries, while enforcing global mass conservation by rectifying of the outlet velocity components. It is shown in Fig.1(b). Fig.1(c) illustrates computational grids around the axisymmetric ogive. The mesh for the hydrofoil comprises $485 \times 80$ points. The mesh distribution is chosen to facilitate adequate resolution of cavitation zone.

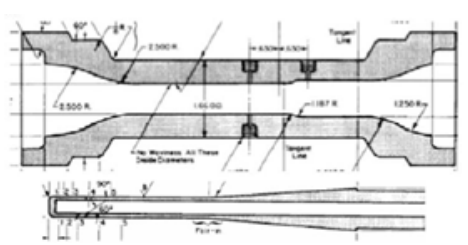

(a)

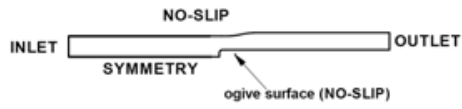

(b)

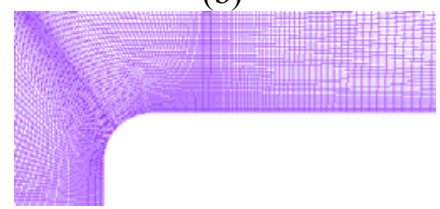

(c)

Fig. 1 The schematic diagram of the dynamic measure system

The two important parameters, the cavitation number and the coefficient pressure are given below:

$$
\begin{gathered}
\sigma=\frac{p_{\infty}-p_{v \infty}}{\frac{1}{2} \rho_{l} U_{\infty}{ }^{2}} \\
C p=\frac{p-p_{\infty}}{\frac{1}{2} \rho_{l} U_{\infty}{ }^{2}}
\end{gathered}
$$

\section{Results and Discussions}

In order to verify the numerical model and the mesh, the boundary conditions are accordant with Hord's experiment. Since considerable blockage effects are present, it became necessary to model details of the tunnel geometry in the simulations. To verify these tunnel interaction effects, we performed single-phase, noncavitating simulations and have compared it to Hord's noncavitating data as shown in Fig.2.

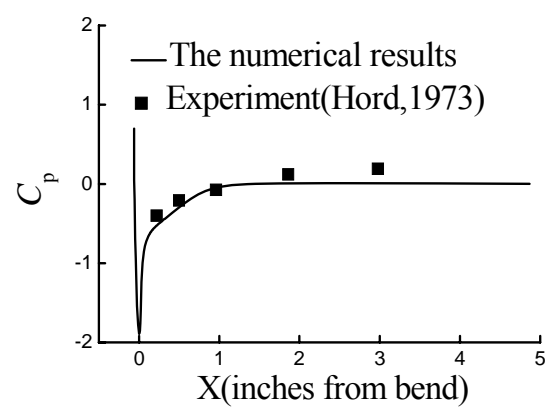

Fig. 2 Comparison the pressure coefficient by calculation and the experiment

In cavitation cases, the cavitation model parameters in water are inadequate in cryogenic fluids, and more appropriate model parameters are needed to calibrate. Fig. 3 portrays the different cavitation model parameters, which demonstrate that the case produces the least RMS error between the computed surface temperature/pressure coefficient. The experimental data when $C_{\text {prod }}$ is 20 and $C_{\text {dest }}$ is 3.8 in liquid Nitrogen. As a result, we hereafter employ these values for the Merkle cavitation Model in liquid 
nitrogen. Following our assessment with liquid Nitrogen, we extend our focus to the cases with liquid Hydrogen. We experience the need to re-calibrate our cavitation models for this different fluid. The cavitation model parameters become 7 and 2.1 for $C_{\text {prod }}$ and $C_{\text {dest }}$ respectively.
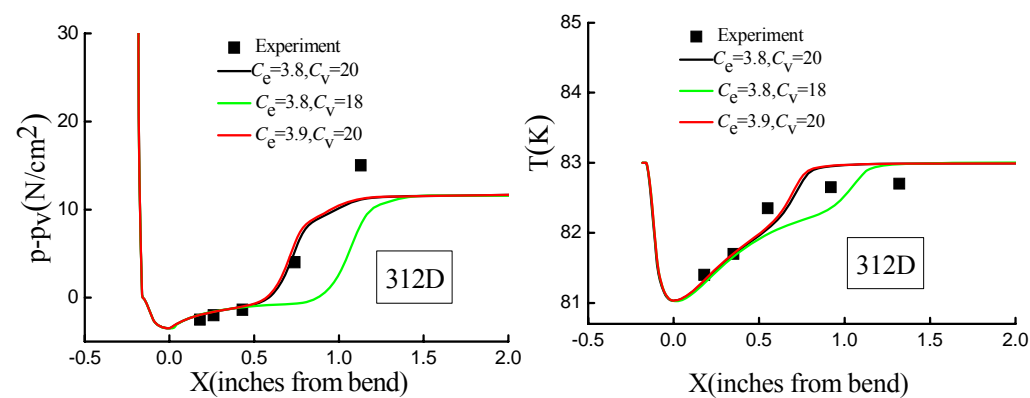

Fig. 3 Comparisons of pressure and temperature depression with different cavitation model coefficients to the experiment's results

To understand the impact of thermal effects at operating temperatures close to the critical temperature of the fluid, we analyze the liquid nitrogen and hydrogen property firstly. The critical temperature of nitrogen is $126.19 \mathrm{~K}$ and the triple point is at $63.15 \mathrm{~K}$. The variation of nitrogen properties along the saturation line for liquid and vapor densities as well as the vapor pressure are shown in Fig. 4 for an operating range of $70-100 \mathrm{~K}$. The triple point of hydrogen is $13.957 \mathrm{~K}$ and the critical temperature is $33.19 \mathrm{~K}$. The entire operational range of liquid hydrogen systems is only about $19 \mathrm{~K}$ which is shown in Fig.5. Tab.1 presents the range of the property for nitrogen and hydrogen.
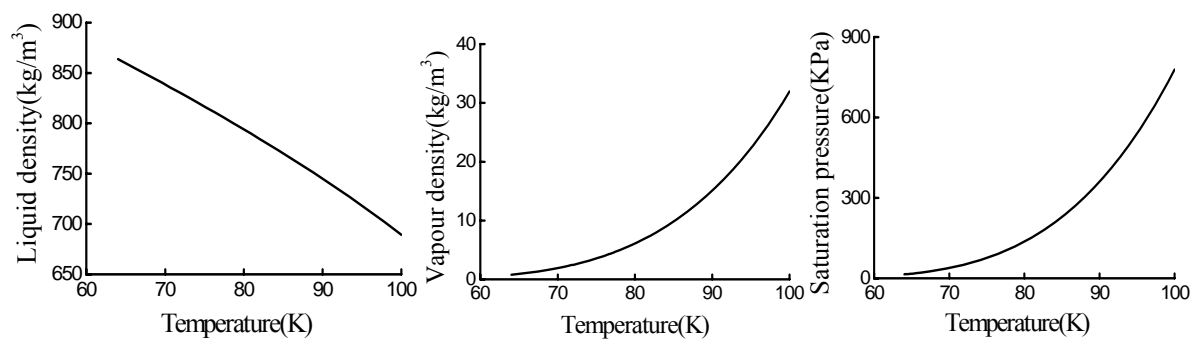

Fig. 4 Saturation properties of nitrogen as a function of temperature
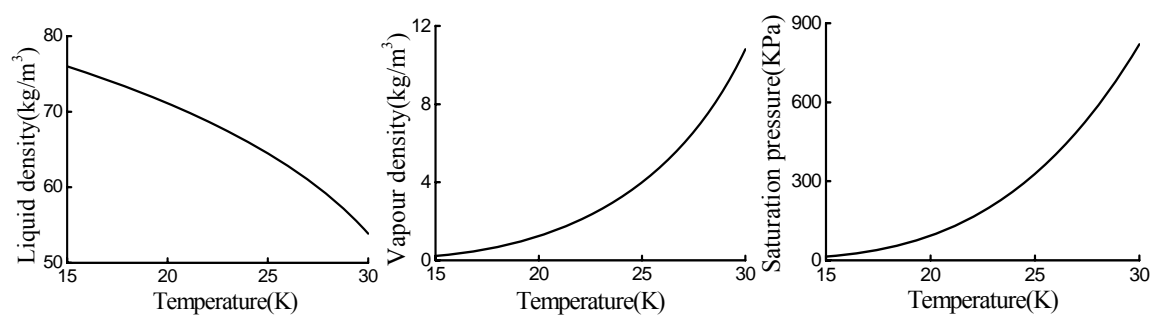

Fig. 5 Saturation properties of hydrogen as a function of temperature

Table 1 The range property of liquid nitrogen and hydrogen

\begin{tabular}{c|c|c|c|c|c}
\hline \hline Material & $\begin{array}{c}\text { Liquid } \\
\text { density } \\
\left(\mathrm{kg} \mathrm{m}^{\wedge}-3\right)\end{array}$ & $\begin{array}{c}\text { Vapour } \\
\text { density } \\
\left(\mathrm{kg} \mathrm{m}^{\wedge}-3\right)\end{array}$ & $\begin{array}{c}\text { Vapour pressure } \\
(\mathrm{Pa})\end{array}$ & $\begin{array}{c}\text { Latent heat } \\
\left(\mathrm{J} \mathrm{kg}^{\wedge}-1\right)\end{array}$ & $\begin{array}{c}\text { Operational } \\
\text { temperature } \\
\text { range }(\mathrm{K})\end{array}$ \\
\hline $\mathrm{N}_{2}$ & $863-689$ & $0.77-31.9$ & $14602-778274$ & $\begin{array}{c}214623- \\
160975\end{array}$ & $64-100$ \\
\hline $\mathrm{H}_{2}$ & $53.8-76$ & $0.22-10.8$ & $13440-819900$ & $450795-292170$ & $15-30$ \\
\hline
\end{tabular}

Table 2 The calculation conditions for Nitrogen

\begin{tabular}{c|c|c|c|c}
\hline \hline Substance & Case & $\sigma_{\infty}$ & $\mathrm{U}_{\infty}$ & $\mathrm{T}_{\infty}$ \\
\hline $\mathrm{N}_{2}$ & $312 \mathrm{D}$ & 0.46 & 23.5 & $83 \mathrm{~K}$ \\
\hline $\mathrm{N}_{2}$ & $322 \mathrm{E}$ & 0.44 & 26.8 & $88.56 \mathrm{~K}$ \\
\hline $\mathrm{H}_{2}$ & $349 \mathrm{~B}$ & 0.38 & 63.6 & $21.33 \mathrm{~K}$ \\
\hline
\end{tabular}



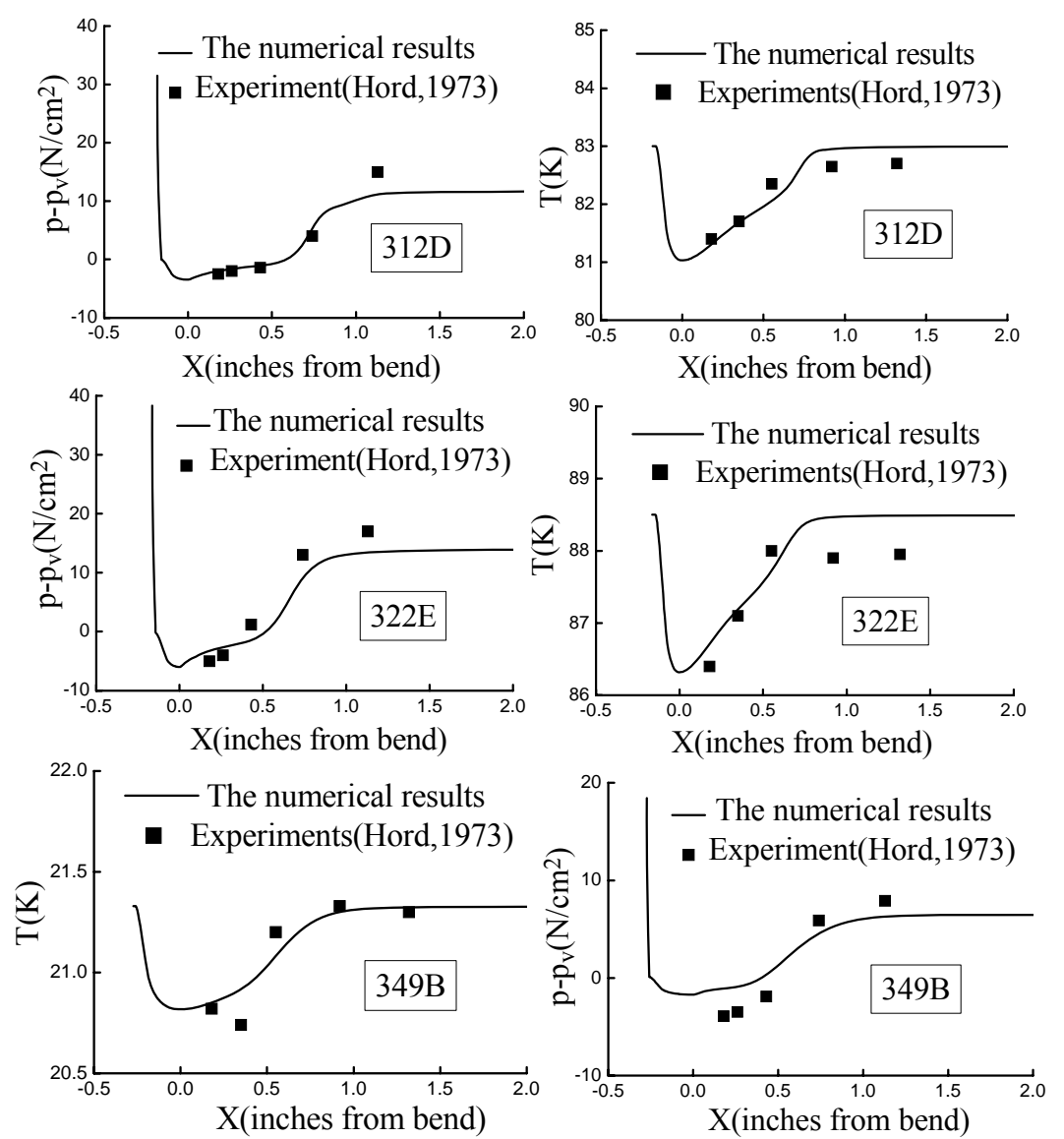

Fig. 6 Comparisons of pressure and temperature depressions in liquid nitrogen to the experiment's results at conditions given in Tab.2.

In order to analyze the thermal effect on cavitation in cryogens, Figure 6 compares the pressure and temperature depressions to the experimental results in liquid nitrogen and hydrogen at three conditions in Tab.2. We firstly note that the numerical models are able to provide a reasonable results from the standpoint of their predictive capabilities with the experimental data. From Fig.6, it is dictated that the temperature depresses due to absorb the latent heat around the cavity region, and the local pressure depresses. From pressure depression in Fig.6, if thermal effects are not considered, this value would be zero in the cavity, while the values below zero in the cryogenic case indicate pressure depression due to thermal effects. It is also observed that a slight temperature rise can be found above the reference fluid temperature at the rear of cavity, which is attributed to the release of latent heat during the condensation process. It further illustrates why thermal effects have a substantial impact on the performance of cryogenic pumps. The pressure in the rear of the hydrofoil from the calculation returns to the free-stream pressure faster, but slower for the experiment. This is because the influence of the re-entrant jet and the energy non-equilibrium due to energy dissipation and other factors are large.

Our contention on the thermal effect is corroborated by the local cavitation number depicted in Fig.7. The freestream cavitation number $\left(\sigma_{\infty}\right)$ of the $322 \mathrm{E}$ case is smaller than the 312D case (Tab.2). Thus, under isothermal conditions, an increase in the cavity length is expected from case $312 \mathrm{D}$ case to $322 \mathrm{E}$ case. However, the combination of evaporative cooling and its resultant impact over the vapor pressure causes a sharp increase in the effective cavitation number closed to the cavitation zone. This increase is more substantial for the $322 \mathrm{E}$ case and eventually leads to comparable levels of effective cavitation number between the two cases, as seen in Fig.7. On the contrary, the surface pressure plots in Fig. 6 clearly indicate a decrease in cavity length from $312 \mathrm{D}$ case to $322 \mathrm{E}$ case, despite the decrease in the freestream cavitation number. This fact clearly distills the significant impact of the thermal effect in cryogenic fluids, especially under working conditions that are close to the thermodynamic critical point.

The maximum pressure and temperature depression is also an important evaluation methods. The maximum pressure is described as $\left(p_{v \infty}-p_{\min }\right) / p_{v \infty}$. For 312D case, the maximum pressure depression relative to the freestream saturation pressure is about $18.5 \%$ and the maximum temperature depression is about $1.97 \mathrm{~K}$, but for $322 \mathrm{E}$ case, the maximum pressure depression is about $21.6 \%$ and temperature depression is about $2.18 \mathrm{~K}$. 


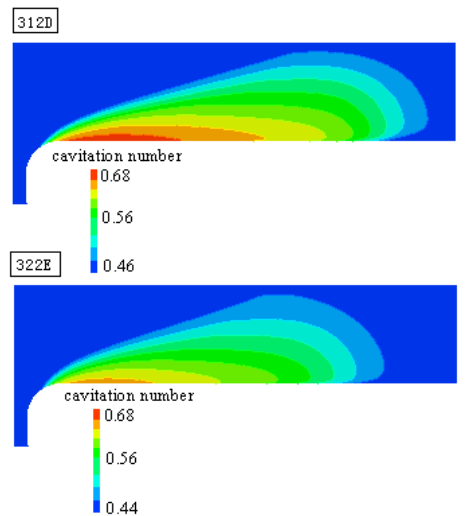

Fig. 7 The local cavitation number for $312 \mathrm{D}$ and $322 \mathrm{E}$ cases $\left(\sigma=p_{\infty}-p_{v}\left(T_{c}\right) / 0.5 \rho_{l} U_{\infty}{ }^{2}\right)$

In Fig.8, as a further assessment of the influence of thermal effect on cryogenic cavitation based on Case 312D, we compare the present cryogenic model solution with the isothermal solution, obtained by using the identical model except that the energy equation is not invoked and the property of the nitrogen is at a constant value. Clearly, the thermal field does affect the cavity structures. For the isothermal case, a sharp and distinct cavity is obtained with vapor volume fraction in the cavity being near unity. As the thermal effect is considered, the cavity interface becomes less sharp and the vapour volume fraction in the cavity drops dramatically, and the maximum vapour volume fraction is approximately 0.7 at the leading edge and it is being even smaller in the interior. The reason of the porous cavity is that water vapour volume fraction is small, so the mixture density increases, caused the gradient of the density of the liquid-vapour interface becomes smaller. This also indicates that the bulk of the vaporization is occurring near the leading edge with subsequent vaporization downstream, cavitation is suppressed due to the temperature depression at the leading edge, it shows increased spreading while getting shorter in length, compared with the isothermal case, as shown in Fig.8(a)、(c) and (d). Fig.8(b) presents the temperature distribution, from it we can see that in the cavity region, the temperature depresses due to absorb the evaporation latent heat, and when the vapour volume fraction is higher, the temperature depression and pressure depression becomes bigger. The maximum temperature depression is close to the leading edge of the ogive which from reference temperature $83 \mathrm{~K}$ reduced to $81.03 \mathrm{~K}$. Finally, in Fig.8(e), the pressure profile inside the cavity is steeper under the cryogenic condition than that under the isothermal condition. For the isothermal case, the pressure in the cavity is at a constant value which equal to the local vapor pressure. However, with considering the thermal effect, there is depression at the leading edge due to local temperature drop.

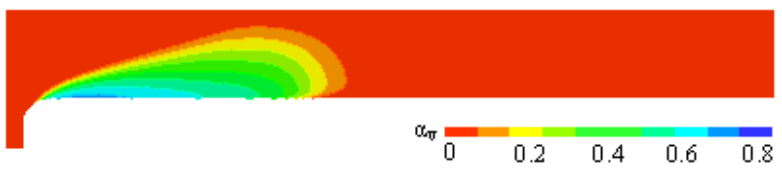

(a) $\alpha_{v}$ for the thermal effects case

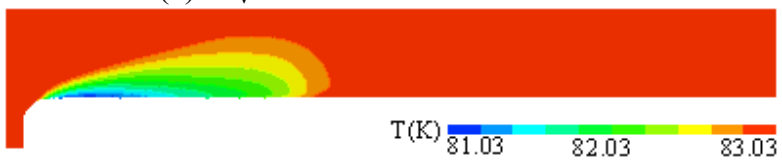

(b) The temperature distribution

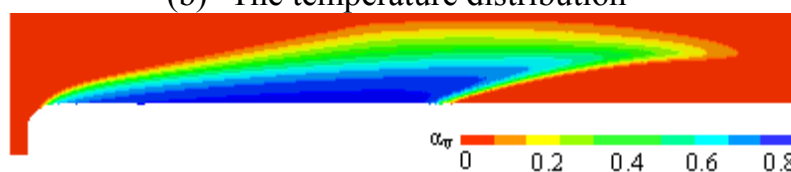

(c) $\alpha_{v}$ for the isothermal case

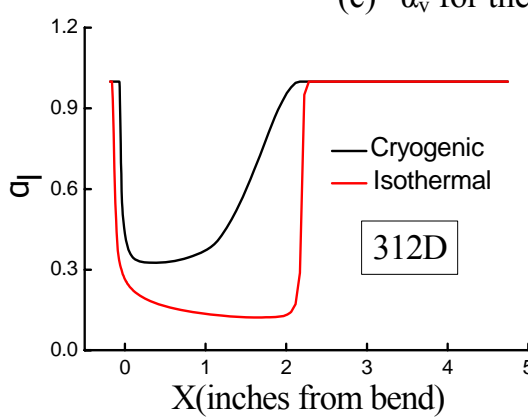

(d) $\alpha_{1}$ along surface

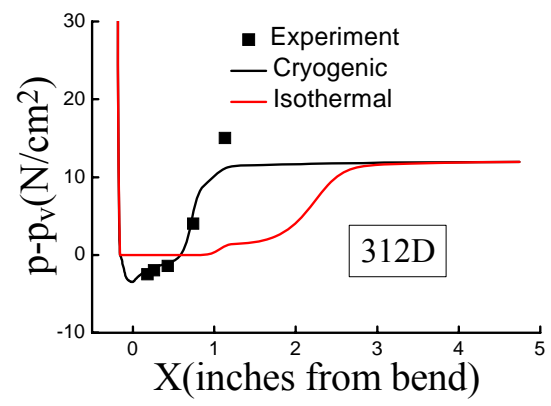

(e) pressure along surface

Fig. 8 Comparisons the results for 312D Case between the isothermal and thermal effect case

In order to investigate the intensity of thermal effect on cavitation in different fluids. The 349B case is clarified the thermal effect in liquid hydrogen. The trends for hydrogen are in general similar to what is discussed for liquid nitrogen. In cavity region, the saturation pressure and temperature depresses. Furthermore, the discrepancy in our predictions for liquid hydrogen, when subjected to higher velocities, is also evidenced by the plots for the case 349B. Overall, the agreement with experimental data in 
the case 349 is better in terms of the surface temperature than pressure. The pressure profiles inside the cavity and particularly in the closure regions are more inconsistent and showing larger differences, but it is better than the results of Cao ${ }^{[10]}$. Fig. 6 shows that the maximum pressure depression is $22.54 \%$, and the maximum temperature depression is $0.51 \mathrm{~K}$. It dictates that the mean level of the maximum pressure depression for $1 \mathrm{~K}$ is much higher for liquid hydrogen than that for liquid nitrogen calculations. This is due to the difference of the material property, the intensity of thermal effect on the cavitating flows for the two kinds of fluids appears some distinction. From Fig.4 and Fig.5, we can see the variation of the two fluids with temperature is general similar. Combined with Tab.1, it is obtained that the density ratio is smaller for hydrogen than it is for nitrogen, in order to obtain the same size cavity, it needs more mass to evaporate in hydrogen. The slope of vapor pressure variation for hydrogen is about twice as high as liquid nitrogen, and it is approximately $30 \mathrm{KPa} / \mathrm{K}$ for hydrogen in $20.4 \mathrm{~K}$ as compared to $16 \mathrm{KPa} / \mathrm{K}$ for liquid nitrogen in $81 \mathrm{~K}$. Hence thermal effects are more pronounced in hydrogen.

\section{Conclusions}

Numerical simulations of cavitation in liquid nitrogen and liquid hydrogen over an axisymmetric ogive are conducted respectively. The thermal effects on cavity characteristics are discussed. Based on the study, some conclusions are obtained:

(1) The thermal effect more pronounced is shown in that the vapour content is far less, and the cavity becomes more porous and the cavity shows increased spreading while getting shorter in length.

(2) In the cavity region, the temperature around the forepart of cavity depresses due to absorb the evaporation latent heat, and the local saturation pressure drops. At the rear of cavity, the temperature rises attributed to the release of latent heat during the condensation process.

(3) When the fluid is operating close to its critical temperature, thermal effects on cavitation are more obviously in both the liquid nitrogen and hydrogen. The thermal effect in liquid hydrogen is more distinctively than it is in liquid nitrogen attributed to the changes of density ratio, vapour pressure and other variable properties of the fluid.

\section{Acknowledgments}

The authors gratefully acknowledge support by the National Natural Science Foundation of China (NSFC, Grant No.: 50979004) and Doctor Reserch Fund of Univercity(Grant No.: 20080070027).

\section{Nomenclature}

$\begin{array}{llll}P_{\infty} & \text { Freestream static pressure in inlet }[\mathrm{Pa}] & U_{\infty} & \text { Freestream velocity [m/s] } \\ \dot{m}^{+} & \text {Eevaporation rates } & C_{\text {prod }} & \text { Evaporation rates coefficients } \\ \dot{m}^{-} & \text {Condensation rates } & C_{d e s t} & \text { Condensation rates coefficients } \\ \rho_{l} & \text { The liquid density[kg m³] } & P_{V \infty} & \text { Freestream saturation pressure [Pa] } \\ v & \text { Liquid dynamic viscosity coefficient } & \rho_{m} & \left.\text { The mixture density[ } \mathrm{kg} \mathrm{m}^{\wedge} 3\right] \\ P & \text { Pressure[Pa] } & \mu & \text { The laminar viscosity } \\ u & \text { Velocity }[\mathrm{m} / \mathrm{s}] & \mu_{t} & \text { The turbulent viscosity } \\ L & \text { Latent heat }[\mathrm{J} \mathrm{kg} \wedge-1] & \operatorname{Pr}_{L} & \text { The laminar Prandtl number } \\ H & \text { Enthalpy[J kg^-1] } & \operatorname{Pr}_{t} & \text { The turbulent Prandtl number } \\ f_{v} & \text { Mass volume fraction } & P_{k} & \text { Turbulent production } \\ i, j, k & \text { The axes directions, respectively } & P_{v}\left(T_{l}\right) & \text { Local saturation pressure[Pa] } \\ \alpha_{l} & \text { Water volume fraction } & P_{V \infty} & \text { Freestream saturation pressure }[\mathrm{Pa}] \\ p_{v} & \text { The phase-change threshold pressure[Pa] } & & \end{array}$

\section{Reference}

[1] Utturkar, Y., Wu, J., Wang, G., Shyy, W., 2005, "Recent progress in modeling of cryogenic cavitation for liquid rocket propulsion," Progress in Aerospace Sciences, Vol. 41, pp. 558-608.

[2] Sarosdy, L.R. and Acosta, A.J., 1961, "Note on Observations of Cavitation in Different Fluids," Paper 60-WA-83, ASME Winter Annual Meeting, New York.

[3] Hord, J., 1973b, “Cavitation in Liquid Cryogens,” III - Ogives, NASA Contractor Report, NASA CR - 2242.

[4] Franc, J.P., Janson, E., Morel, P., Rebattet, C. and Riondet, M., 2001, "Visualizations of Leading Edge Cavitation in an Inducer at Different Temperatures," Presented at Fourth International Symposium on Cavitation, Pasadena, Canada.

[5] Stahl, H. A., and Stepanoff, A. J., 1956, "Thermodynamic Aspects of Cavitation in Centrifugal Pumps," Journal of Basic Engineering, Vol. 78, pp. 1691-1693.

[6] Ruggeri, R.S. and Moore, R.D. 1969, "Method of Prediction of Pump Cavitations Performance for Various Liquids, Liquid Temperatures and Rotation Speeds," NASA Technical Note, NASA TN D-5292.

[7] Holl, J. W., Billet, M. L., and Weir, D. S., 1975, “Thermodynamic Effects On Developed Cavitations," Journal of Fluids Engineering, Vol. 97, No. 4, pp. 507-516.

[8] Hosangadi, A. and Ahuja, V. 2005, "Numerical Study of Cavitation in Cryogenic Fluids," J. Fluids Engineering, Vol. 127, pp. 267-281.

[9] Utturkar Y, Thakur S, Shyy W. 2005, “Computational modeling of thermodynamic effects in cryogenic cavitation," In: Proceedings of the 43rd AIAA Aerospace Sciences Meeting and Exhibit, Reno, NV, United States. 
[10] Cao X L, Zhang X B, Qiu L M, et al. 2009, "Validation of full cavitation model in cryogenic fluids," Chinese Sci Bull, Vol. 54, No. 10, pp. 1633-1640.

[11] Florian R.Menter. "Zonal Two Equation $k-\omega$ Turbulence Models for Aerodynamic Flow," $24^{\text {th }}$ Fluild Dynamics Conference.

[12] Strelets M. 2001, "Detached eddy simulation of massively separated flows," AIAA-01-0879.

[13] Merkle C L, Feng J, Buelow P E O. 1998, “Computational modeling of dynamics of sheet cavitations," In: Proceedings of the 3rd International Symposium on Cavitation, Grenoble, France.

[14] Singhal, A. K., Athavale, M. M. 2002, "Mathematical Basis and Validation of the Full Cavitation Model," Journal of Fluids Engineering, Vol. 124, pp. 617-624. 